\title{
ESTRUTURA POPULACIONAL E ESPACIAL DE Cereus jamacaru DC. EM DUAS ÁREAS DE CAATINGA DO AGRESTE DA PARAÍBA, BRASIL
}

\author{
POPULATIONAL AND SPATIAL STRUCTURE OF Cereus jamacaru DC. IN TWO AREAS OF \\ 'CAATINGA' BIOME IN PARAÍBA STATE, BRAZIL
}

\author{
Alex da Silva Barbosa ${ }^{1}$ Alberício Pereira de Andrade ${ }^{2}$ Lécio Resende Pereira Júnior ${ }^{3}$ \\ Riselane de Lucena Alcântara Bruno ${ }^{4}$ Robson Luís Silva de Medeiros ${ }^{5}$ \\ Miguel Avelino Barbosa Neto ${ }^{5}$
}

\begin{abstract}
RESUMO
As cactáceas nativas ainda constituem um grupo de plantas que necessita de estudos sobre sua ocorrência, distribuição e dinâmica para subsidiar ações de manejo e conservação da família na região de inserção. $\mathrm{O}$ objetivo deste estudo foi analisar a estrutura e distribuição espacial de Cereus jamacaru DC. em uma mata de cipó (município de Campina Grande) e em uma caatinga arbórea (município de Monteiro) no semiárido da Paraíba, Brasil. Foram plotadas 100 parcelas com dimensões de $10 \mathrm{~m}$ x $10 \mathrm{~m}$ em cada área. Dentro das parcelas, todos os indivíduos foram etiquetados e mensurados na circunferência da base do caule com auxílio de uma fita métrica e a altura total com auxílio de tubos de PVC graduados. A estrutura espacial das populações foi obtida pela coleta da coordenada geográfica de cada indivíduo em cada área e expressa por meio de mapas de distribuição espacial. Foram amostrados 39 espécimes em Campina Grande, apresentando área basal (AB) de $0,69 \mathrm{~m}^{2}$, densidade absoluta (DA) de 39 ind. ha ${ }^{-1}$, frequência absoluta (FA) de $32 \% \mathrm{e}$ tendência ao agrupamento (IGA=1,01). Em Monteiro, foram amostrados 38 indivíduos, $A B=0,47 \mathrm{~m}^{2}$, $\mathrm{DA}=38$ ind. $\mathrm{ha}^{-1}, \mathrm{FA}=29 \%$ e tendência ao agrupamento $(\mathrm{IGA}=1,11)$. Os indivíduos de $C$. jamacaru apresentam padrão de distribuição agrupado em ambas as caatingas secundárias, sendo que a população de Monteiro enfrenta problemas de recrutamento, o que poderá implicar no insucesso reprodutivo da população futura. A espécie apresenta padrão de distribuição similar a outras cactáceas colunares.
\end{abstract}

Palavras-chave: demoecologia; Cactaceae; mandacaru.

\section{ABSTRACT}

The native cacti still constitute a group of plants that require data in relation to its occurrence, distribution and dynamics with vegetation to subsidize management and conservation of families in the region. The aim of the study was to analyze the structure and the spatial distribution of Cereus jamacaru in a hypoxerophytic caatinga (In Campina Grande) and in a hyperxerophilic caatinga (In Monteiro) areas of semiarid of Paraíba state, Brazil. It had been plotted 200 parcels with $10 \mathrm{mx} 10 \mathrm{~m}$ in the municipalities of Campina Grande and Monteiro, PB state. In each parcel, all the individuals were sampled and labeled by measuring with the aid of tape measuring the base circumference of each specimen and height was earned with graduates PVC

1 Licenciado em Ciências Agrárias, MSc., Doutorando do Programa de Pós-Graduação em Agronomia, Universidade Federal da Paraíba, Rodovia PB 079 - Km 12, CEP 58397-000, Areia (PB), Brasil. aldasibarbosa@cchsa.ufpb.br

2 Engenheiro Agrônomo, Dr., Programa de Pós-Graduação em Zootecnia, Universidade Federal da Paraíba, Rodovia PB 079 - Km 12, CEP 58397-000, Areia (PB), Brasil. albericio@uol.com.br

3 Engenheiro Agrônomo, MSc., Doutorando do Programa de Pós-Graduação em Ecologia e Recursos Naturais, Universidade Federal do Cerará, Centro de Ciências - Bloco 902, CEP 60455-970 - Fortaleza-CE, Brasil. leciojunior@hotmail.com

4 Engenheiro Agrônomo, Dr $^{\mathrm{a}}$., Programa de Pós-Graduação em Agronomia, Universidade Federal da Paraíba, Rodovia PB 079 - Km 12, CEP 58397-000, Areia (PB), Brasil. lanebruno.bruno@gmail.com

5 Licenciado em Ciências Agrárias, Universidade Federal da Paraíba, Campus de Bananeiras, CEP 58220-000, Bananeiras (PB), Brasil. robsonluissm@hotmail.com/miguelavelinoneto18@gmail.com

Recebido para publicação em 3/04/2014 e aceito em 13/04/2015

Ci. Fl., v. 27, n. 1, jan.-mar., 2017 
pipes. For evaluation of spatial structure, we obtained coordinates of each individuals, then, the maps of spatial distribution was produced. Thirty-nine 39 specimens were sampled in Campina Grande, showing basal area (BA) of $0.69 \mathrm{~m}^{2}$, absolute density (AD) of $39 \mathrm{ind}^{-1} \mathrm{ha}^{-1}$, the absolute frequency (AF) of $32 \%$ and a tendency to cluster ( $\mathrm{IGA}=1,01$ ). In Monteiro, 38 individuals were sampled, $\mathrm{AB}=0.47 \mathrm{~m}^{2}, \mathrm{AD}=38$ ind. $\mathrm{ha}^{-1}, \mathrm{AF}=29 \%$ and a tendency to cluster (IGA $\left.=1,11\right)$. The specimens of Cereus jamacaru showed clustering distribution pattern in the areas of secondary Caatinga, being that Monteiro's population faces recruitment problems. Thus, it could imply in reproductive unsuccessful population future. The species shows similar distribution pattern in relation to other columnar cacti.

Keywords: demoecology; Cactaceae; mandacaru.

\section{INTRODUÇÃO}

A família Cactaceae compreende 124 gêneros e 1.438 espécies (HUNT et al., 2006), configurando como a segunda maior família botânica endêmica das Américas, logo depois da Bromeliaceae (TAYLOR; ZAPPI, 2004). O Brasil é considerado o terceiro maior centro de diversidade da família Cactaceae, com um total de aproximadamente 200 espécies (SOUZA; LORENZI, 2005). A família encontra-se subdividida em três subfamílias: Opuntioideae, Cactoideae e Pereskioideae (NYFFELER, 2002).

As Cactaceae ocorrem entre as latitudes a $35^{\circ}$ Norte e Sul, ao nível do mar até mais de $5000 \mathrm{~m}$ de altitude (BOYLE; ANDERSON, 2002; TAYLOR; ZAPPI, 2004). Seus espécimes possuem a capacidade de crescer nas mais diferentes fitofisionomias, entretanto, a maior diversidade é encontrada em regiões áridas e semiáridas nas quais atuam com recursos importantes para vertebrados e invertebrados principalmente na estação seca.

O mandacaru (Cereus jamacaru DC.) pertence ao gênero Cereus que possui aproximadamente 34 espécies, pertence à subfamília Cactoideae, tribo Cereeae. As espécies deste gênero são de porte arbustivoarbóreo, plantas de hábito de crescimento colunar, caule com conformação angular caracterizado por apresentar grandes aréolas com formações espinhosas e antese noturna (ANDERSON, 2001). Os frutos do mandacaru constituem como um importante recurso para diferentes grupos de animais como répteis, pássaros, insetos e mamíferos, para além de ser utilizado como alimento para o gado bovino

(GOMES et al., 2014; CAVALCANTI, RESENDE, [s. d.]).

Diversos trabalhos abordando padrões de distribuição de cactáceas já foram realizados (HERNÁNDEZ; BÁRCENAS, 1995; MOURELLE; EZCURRA, 1997; GUERRERO et al., 2008; SANTOS, 2009), entretanto, ainda são poucos os trabalhos realizados com as espécies da caatinga nordestina. Neste sentido, as cactáceas nativas ainda constituem um grupo de plantas que necessita de estudos que venham elencar dados de sua ocorrência, distribuição, diversidade e dinâmica, como premissas para o estabelecimento de programas de manejo e conservação da família na região de inserção.

Observações realizadas por Ribeiro (2011) em áreas de caatinga em sucessão secundária média, demonstraram que o gênero Pilosocereus (Cactaceae) podem exercer uma relação de dominância e oportunismo nestes ambientes. Neste sentido, estudos similares são importantes para compreender melhor os nichos desses táxons em ecossistemas naturais com diferentes estágios de sucessão ecológica.

Portanto, o objetivo deste estudo foi analisar a estrutura horizontal e espacial de Cereus jamacaru em uma caatinga hipoxerófila (município de Campina Grande) e em caatinga hiperxerófila (município de Monteiro) no semiárido da Paraíba, Brasil.

\section{MATERIAL E MÉTODOS}

\section{Áreas de estudo}

A área de caatinga de Campina Grande - PB, objeto deste estudo, pertence ao Instituto Nacional do Semiárido (INSA) - (7¹6’35,97’S 3557’55,72”O) (Figura 1). O município de Campina Grande está localizado na Mesorregião do Agreste Paraibano, com uma área de $621 \mathrm{~km}^{2}$. A sede do município possui altitude de 551 metros distando $112 \mathrm{~km}$ da capital João Pessoa (COMPANHIA DE PESQUISA 
DE RECURSOS MINERAIS, 2005b). Segundo informações de moradores da região, a área foi utilizada há 25 anos para os cultivos de algodão (Gossypium hirsutum L.) e agave (Agave sisalana Perrine). A área apresenta um mosaico de diferentes classes de solos, sendo predominantes os Argissolos (REINALDO; SILVA, 2006), constituídos por material mineral, apresentando horizonte B textural imediatamente abaixo do A ou E, com argila de atividade alta conjugada com saturação por bases baixa e/ou caráter analítico na maior parte do horizonte B (EMBRAPA, 2006). A vegetação predominante é formada pela Mata de Cipó (IBGE, 2012), que se modifica drasticamente ao longo das estações seca e chuvosa. O clima de acordo com a classificação de Köppen é do tipo As, quente e úmido com chuvas de outono-inverno. A estação chuvosa se inicia entre janeiro e fevereiro com término em setembro, podendo se adiantar até outubro, com precipitação média anual de $800 \mathrm{~mm}$. (COMPANHIA DE PESQUISA DE RECURSOS MINERAIS, 2005a).

O trecho de caatinga estudado em Monteiro - PB é de propriedade particular, denominada Sítio Mocó de Baixo ( $7^{\circ} 10^{\prime} 23,1^{\prime \prime} S$ 36 09'53,4”O) (Figura 1). O município de Monteiro ocupa uma área de $986 \mathrm{~km}^{2}$, com altitude aproximada de 599 metros distando $263 \mathrm{~km}$ da capital João Pessoa (COMPANHIA DE PESQUISA DE RECURSOS MINERAIS, 2005b). O clima é do tipo BSh, semiárido quente, com chuvas de verão, segundo a classificação de Köppen, com precipitação pluviométrica média de $431,8 \mathrm{~mm}$ anual. As médias de temperatura dificilmente são inferiores a $24^{\circ} \mathrm{C}$. O tipo de solo predominante na região são os Luvissolos, que compreendem solos minerais, não hidromórficos, caracterizados pela presença de horizonte B textural com argila de atividade alta e alta saturação por bases, imediatamente abaixo de qualquer tipo de horizonte A (EMBRAPA, 2006). A vegetação da área é composta por uma caatinga arbórea (IBGE, 2012), com trechos de floresta caducifólia (COMPANHIA DE PESQUISA DE RECURSOS MINERAIS, 2005b). A área ao longo dos anos vem sendo mantida como reserva da vegetação, no entanto, cortes seletivos de madeira vêm sendo feitos, bem com a eventual entrada de gado bovino durante a estação seca do ano.

A área de Monteiro apresentou melhor estado de conservação da vegetação nativa, precipitação variável e solo de textura franco-argiloarenosa com elevado teor de cálcio e sódio, não sendo considerados como fatores abióticos limitantes ao crescimento e desenvolvimento de Cereus jamacaru (BARBOSA, 2011).

As duas áreas de estudo foram escolhidas por apresentarem características de formações florestais de caatinga em diferentes níveis de sucessão ecológica e também devido ao registro prévio da ocorrência natural de Cereus jamacaru em ambas. Outro critério importante para a escolha das áreas de estudo foi a necessidade de pesquisar in loco dados inerentes à vegetação na qual ocorre naturalmente a espécie com finalidade de subsidiar informações sobre a ecologia de cactáceas na região.

\section{Determinação da estrutura horizontal e vertical}

O método para amostragem dos indivíduos foi o de parcelas contíguas (MULLER-DOMBOIS; ELLEMBERG, 1974; RODAL et al., 1992; RAMALHO, 2008; BARBOSA, 2011). Em cada área de estudo foram instaladas 100 parcelas de $10 \mathrm{~m}$ x $10 \mathrm{~m}$, totalizando uma área amostral de $20.000 \mathrm{~m}^{2}$.

Esse método vem sendo utilizado para o estudo da caatinga e permite inferências mais apropriadas para determinados aspectos da vegetação em estudo (RAMALHO, 2008; BARBOSA 2011).

Em cada parcela foram amostrados e etiquetados com placas de material PET todos os indivíduos da população. Foi mensurada a altura do caule principal com o auxílio de uma vara tubular graduada de todos os indivíduos e a circunferência foi auferida no nível da base (DNB), com auxílio de suta dendrométrica. O processamento dos dados quantitativos da população para obtenção dos valores de estrutura horizontal, foi realizado por meio do programa Microsoft Office ${ }^{\circledR}$ - Excel 2010. A população foi descrita pela densidade absoluta (DA), frequência absoluta (FA), área basal (AB), dominância absoluta (DoA) e índice de agregação de McGuinnes. 


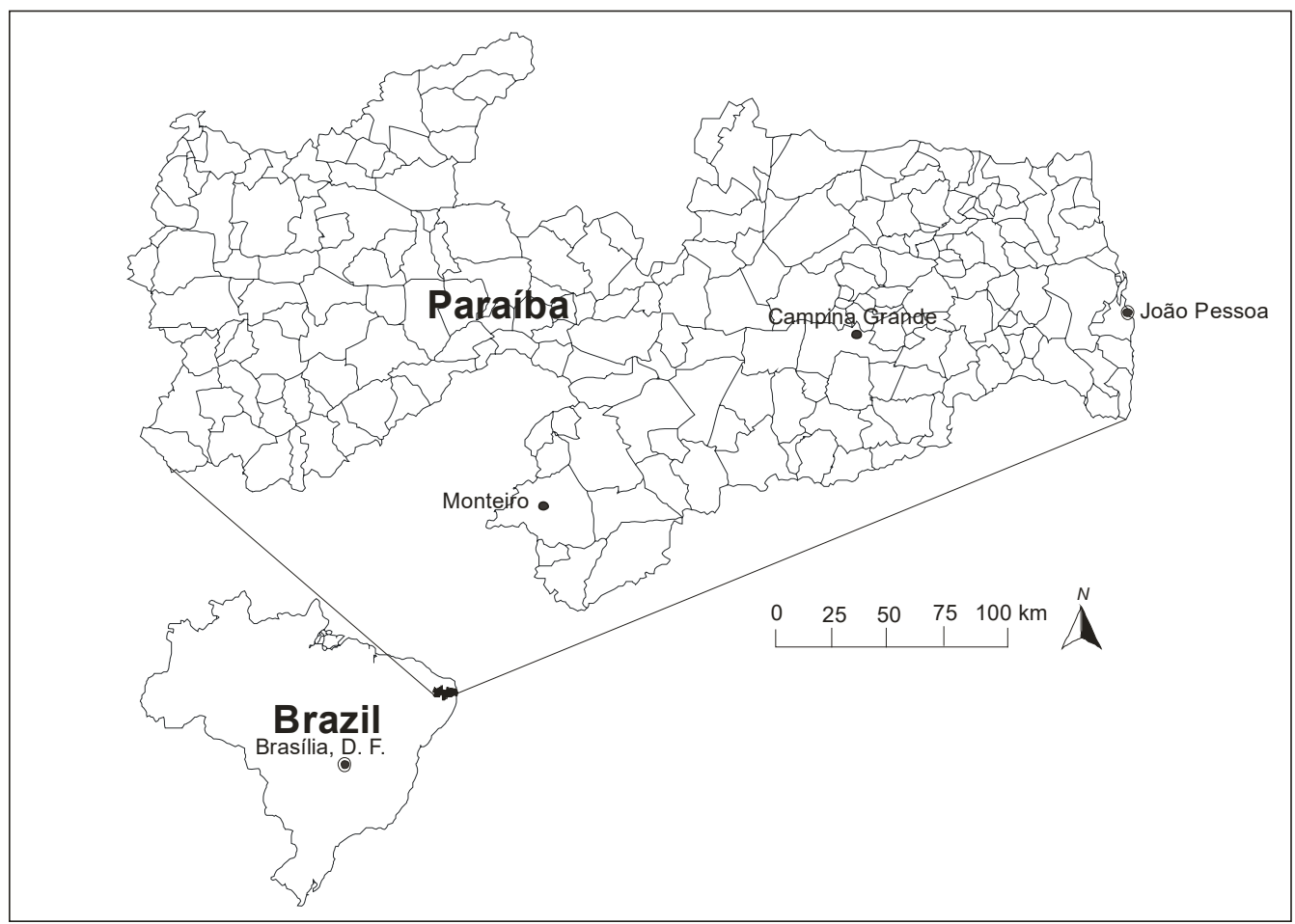

FIGURA 1: Localização dos municípios de Campina Grande e Monteiro - PB, em relação ao estado da Paraíba, Brasil.

FIGURE 1: Location of Campina Grande and Monteiro - PB, in relation to state of Paraíba, Brazil.

\section{Identificação florística da espécie}

Para identificação dos espécimes foram coletadas partes botânicas com presença de descritores, as quais foram acondicionadas em estufa por 48 horas e posteriormente herborizadas. As exsicatas foram enviadas para identificação no Centro de Ciências Agrárias (UFPB/CCA) "Herbário Jayme Coelho de Moraes" (EAN), por meio de comparação com a coleção do herbário. Além disso, foram feitas comparações em literatura especializada. A sinonímia e a grafia do táxon foram atualizadas mediante consulta ao índice de espécies do banco de dados Tropicos ${ }^{\circledR}$ do Missouri Botanical Garden, Saint Louis - Missouri, EUA.

\section{Determinação do índice de agregação da estrutura espacial}

A determinação do índice de agregação ou índice de McGuinnes foi realizada através da equação:

$$
I G A=D / d
$$

Em que: $\mathrm{D}=$ número total de indivíduos da espécie/número total de parcelas alocadas; $d=-\ln (1-F A / 100)(\ln =$ logaritmo neperiano e FA = Frequência Absoluta).

Com auxílio de um GPS Garmin $^{\circledR}$ eTrex Venture ${ }^{\circledR} H C$ foram coletadas as coordenadas de cada indivíduo. O equipamento foi configurado para SAD-69 (Datum Sul-Americano) e o tipo de coordenada foi UTM (Universal Transverse Mercator). As coordenadas foram transferidas para o computador com auxílio do aplicativo MapSource Garmin $^{\circledR}$. Em seguida, os bancos de dados foram montados no Microsoft Office ${ }^{\circledR}$ - Excel 2010.

Os mapas de isolinhas, para a determinação da estrutura espacial em relação ao número de espécimes por parcela, diâmetro do caule e altura, foram produzidos com auxílio dos programas $S U R F E R^{\circledR}$ v. 8 (Golden software, Colorado, EUA) e Corel DRAW ${ }^{\circledR}$ v. X4.

No ambiente do $S U R F E R^{\circledR}$, a conversão dos dados para a plotagem dos mapas foi realizada de 
acordo com o algoritmo da Função de Base Radial (Radial Basis Function) por meio da interpolação de pontos na malha (RAMALHO, 2008). O valor a ser interpolado para qualquer ponto da malha foi estimado por:

$$
G_{j}=\sum_{i=1}^{N_{P T}} w_{i j} Z_{i}
$$

Em que: $\mathrm{G}_{\mathrm{j}}=$ Valor do estimador para o ponto $\mathrm{j} ; \mathrm{N}_{\mathrm{PT}}=$ Número de pontos usados para a interpolação; $\mathrm{Z}_{\mathrm{i}}=$ Valor estimado no ponto $\mathrm{i}$ com valor conhecido; $\mathrm{w}_{\mathrm{ij}}=$ Peso associado ao valor estimado $\mathrm{i}$.

No método de interpolação função de base radial, os pesos são variáveis de acordo com a função básica. A função básica $(B(L))$, neste contexto, é uma função real de $L$ (distância (raio) a partir da origem) (RAMALHO, 2008). Uma das funções mais utilizada é a multiquadrática, representada pela equação:

$$
B(L)=\sqrt{L^{2}+R^{2}}
$$

Em que: B(L) = Função de ponderação do método multiquadrático (CARLSON; FOLEY, 1991); $\mathrm{R}^{2}=$ Parâmetro suavizador especificado pelo usuário.

\section{RESULTADOS}

Foram amostrados 39 (trinta e nove) indivíduos de Cereus jamacaru na caatinga de Campina Grande, na qual a espécie apresentou área basal de $0,69 \mathrm{~m}^{2}$, densidade absoluta de 39 ind. ha ${ }^{-1}$ e frequência absoluta de 32\% nas 100 parcelas amostrais (dados não apresentados). Segundo a classificação do Índice de McGuinnes, os indivíduos apresentaram tendência ao agrupamento ( $\mathrm{IGA}=1,01)$. Na caatinga de Monteiro, foram amostrados 38 (trinta e oito) indivíduos, área basal de $0,47 \mathrm{~m}^{2}$, densidade absoluta de 38 ind. ha ${ }^{-1}$, frequência absoluta de $29 \%$ e tendência ao agrupamento (IGA=1,11) (dados não apresentados). De acordo com a Figura 2 ( $a$ e b), pode-se observar o padrão de tendência ao agrupamento dos indivíduos nas duas áreas. Entretanto, constatou-se, em Campina Grande, formações mais adensadas e bolsões vazios para a espécie na área de Monteiro (Figura $2 \mathrm{a}-\mathrm{b}$ ).

A distribuição da espécie na área de caatinga de Campina Grande demonstrou que $51,1 \%$ das parcelas amostrais foram evidenciados pelo menos 1 indivíduo da espécie em cada parcela, em 9\% delas foram amostrados 2 representantes e 2,4\% 3 representantes desta espécie (Figura 2a). Em Monteiro, em $1,4 \%$ da área foram amostrados 3 espécimes, seguido de 2 plantas $(11,9 \%)$ e 1 planta por unidade amostral correspondendo a maior parte da área (83,4\%) (Figura 2b).

A estrutura hipsométrica da população de Cereus jamacaru na caatinga de Campina Grande ; mostrou-se heterogênea, com uma concentração majoritária de indivíduos na quarta classe $(36 \%$ dos indivíduos), seguida da quinta classe (15\%) e da sexta classe hipsométrica (13\%). As plantas com diâmetro variando entre $6,1-7,0 \mathrm{~m}$ somaram $10 \%$ dos indivíduos. Os espécimes regenerantes somaram 5\% na área (Figura 3a).

Em Monteiro, a espécie apresentou majoritariamente indivíduos concentrados na sexta classe hipsométrica (37\%), seguida da quinta (24\%) e da quarta classe (16\%). Nessa caatinga, os indivíduos encontravam-se mais desenvolvidos quando comparados com a área de Campina Grande (Figura 3b).

$\mathrm{Na}$ área de Campina Grande, a vegetação apresentou espécies indicadoras de processos regenerativos como Mimosa tenuiflora (Fabaceae). Entretanto, em Monteiro, a vegetação demonstrou sinais de melhor conservação, a despeito da existência de focos isolados de degradação. Muitos dos indivíduos de Cereus jamacaru amostrados na segunda área encontravam-se em estágio adulto e, como demonstrado na estrutura hipsométrica, não houve amostragem de indivíduos em fase inicial de crescimento, sendo um impasse para a perpetuação da espécie na área se ações não forem tomadas ações a médio prazo.

Aproximadamente, em 48,5\% da área de Campina Grande foram amostrados espécimes com altura variando de 4 a 5,9 m de altura, em 24,5\% da área observaram-se espécimes com altura de 2 a 3,9 m, e 

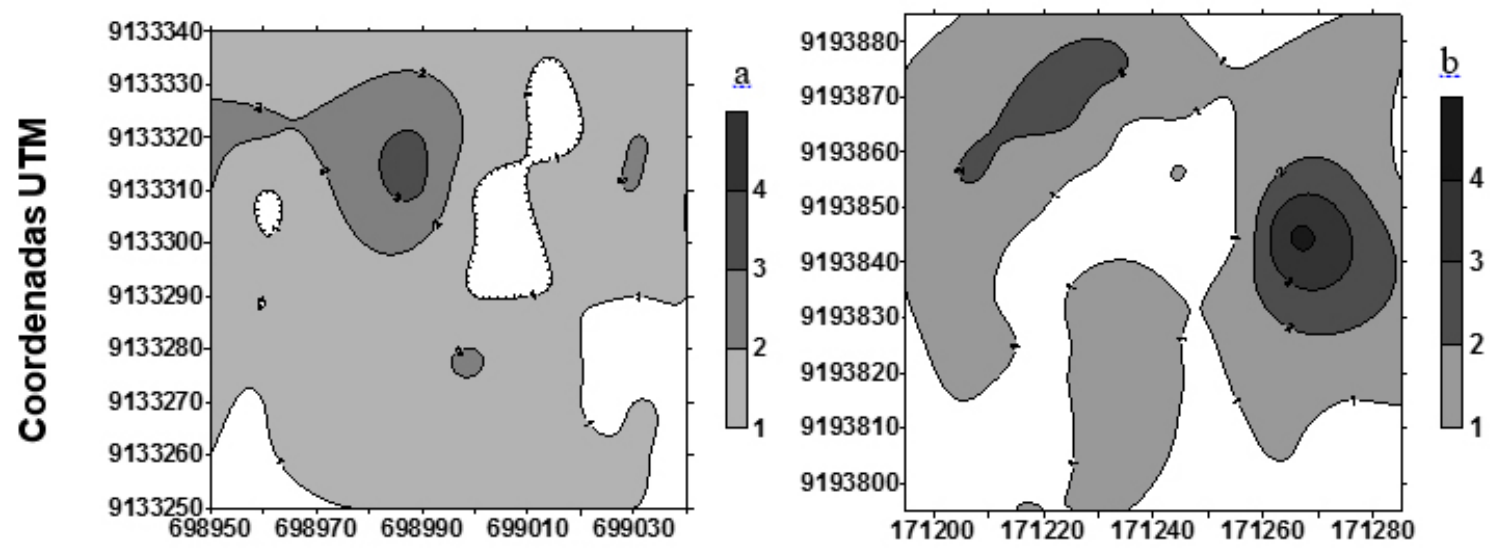

\section{Coordenadas UTM}

FIGURA 2: Mapa de isolinhas de distribuição espacial Cereus jamacaru DC. em duas áreas de caatinga em estágio sucessional intermediário. (a) Área de Campina Grande; (b) Área de Monteiro - PB.

FIGURE 2: Contoured map of spatial distribution Cereus jamacaru DC. in two areas of Caatinga in intermediate successional stage (a) Area of Campina Grande. (b) Area of Monteiro - PB.

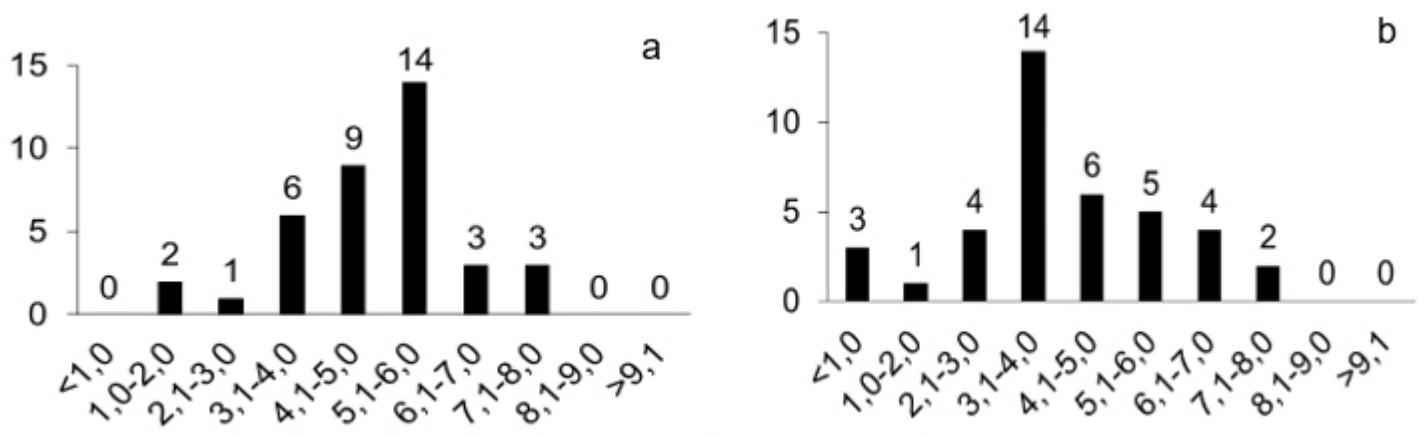

\section{Classe de altura (m)}

FIGURA 3: Estrutura hipsométrica de Cereus jamacaru DC. em duas áreas de caatinga; (a) Campina Grande - PB; (b) Monteiro - PB.

FIGURE 3: Hypsometric structure of Cereus jamacaru DC. in two areas of Caatinga. (a) Area of Campina Grande,PB. (b) Area of Monteiro - PB

plantas com altura variando de 6 a 7,9 m somaram 18,8\% no total. Os indivíduos com altura superior a 8 $\mathrm{m}$ somaram 0,6\% (Figura 4a). Em Monteiro, 42,3\% da área apresentou indivíduos com altura de $6 \mathrm{a} 7,9 \mathrm{~m}$. $21,4 \%$ foi o percentual de plantas com altura igual ou superior a 8 metros e em 13,3\% da área foi registrada a ocorrência de plantas com altura entre 4 e $5,9 \mathrm{~m}$ de altura (Figura $4 \mathrm{~b}$ ).

$\mathrm{Na}$ área Campina Grande, os espécimes apresentaram-se diametricamente concentrados na terceira classe (31\%), seguindos da quarta classe (18\%) (Figura 5a). Em Monteiro, a espécie estudada foi representada por indivíduos com elevado diâmetro. A maior parte dos indivíduos esteve representadada na quinta classe diamétrica (29\%), seguida da quarta (23\%) e terceira classe (16\%) (Figura 5b). Seguindo o padrão da estrutura hipsométrica, não foram amostrados indivíduos na primeira classe de diâmetro. A população apresentou-se adulta, bem como de indivíduos ainda em fase vegetativa que ainda não apresentam antese e, por conseguinte, não apresentaram o desenvolvimento de estruturas reprodutivas para fonecer o recrutamento de novos indivíduos.

De acordo com a Figura 6, pode-se observar a distribuição da espécie por classe de diâmetro. 

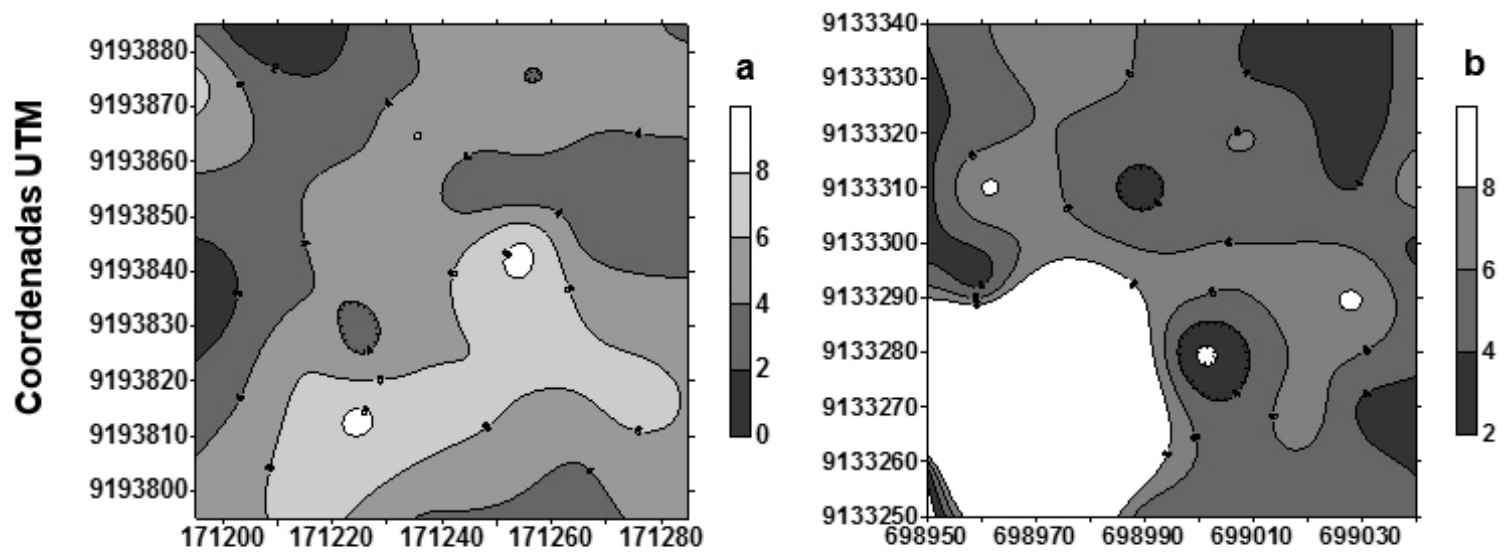

\section{Coordenadas UTM}

FIGURA 4: Mapa de isolinhas de distribuição espacial das classes de altura de Cereus jamacaru DC. em duas áreas de caatinga em estágio sucessional intermediário. (a) Área de Campina Grande; (b) Área de Monteiro - PB.

FIGURE 4: Contoured map of the spatial distribution of height classes of Cereus jamacaru DC. in two areas of Caatinga in intermediate successional stage. (a) Area of Campina Grande. (b) Area of Monteiro - PB.

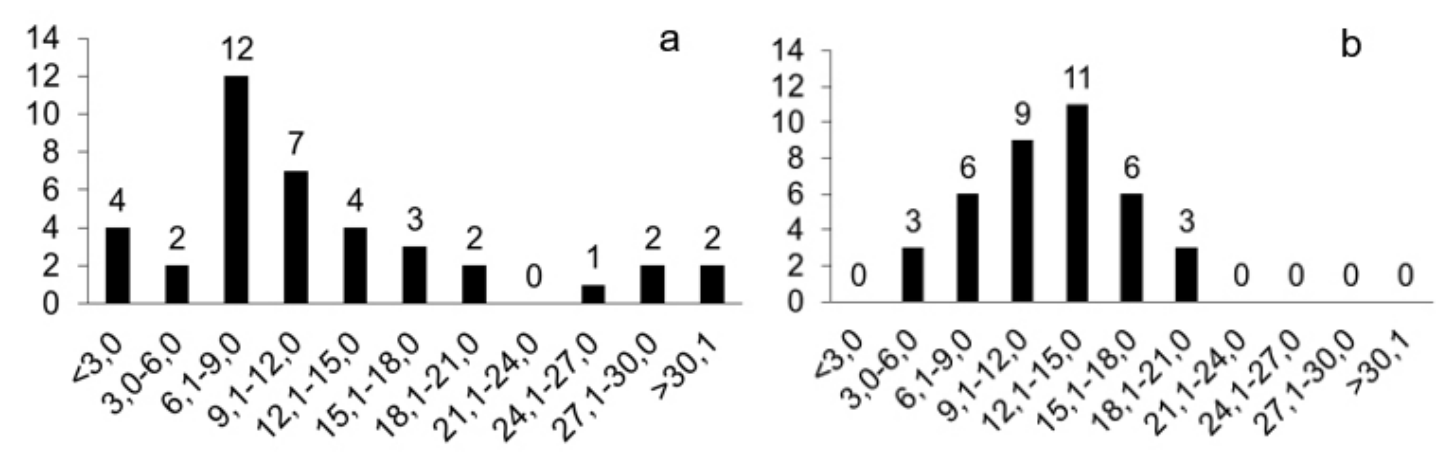

\section{Classe de diâmetro (cm)}

FIGURA 5: Estrutura diamétrica de Cereus jamacaru DC. em uma área de caatinga em estágio sucessional intermediário. (a) Área de Campina Grande; (b) Área de Monteiro - PB.

FIGURE 5: Diameter structure of Cereus jamacaru DC. from Caatinga in intermediate successional stage. (a) Area of Campina Grande. (b) Area of Monteiro - PB

Observa-se que em Monteiro prevaleceram indivíduos mais desenvolvidos, com diâmetros caulinares maiores quando comparados com os espécimes presentes na caatinga de Campina Grande.

Aproximadamente em $42,1 \%$ da área de Campina Grande foram amostrados espécimes com diâmetro ao nível da base (DNB) menor que $1 \mathrm{~cm}$, em 38,4\% da área observaram-se indivíduos com diâmetro variando entre $1,1 \mathrm{~cm}$ a $10 \mathrm{~cm}$. Em 2,5\% da área foram amostradas plantas com diâmetro superior a $20 \mathrm{~cm}$ (Figura 6a). Na caatinga de Monteiro, os espécimes com diâmetros entre 5,1 e $10 \mathrm{~cm}$ somaram $65,3 \%$, e os indivíduos com diâmetros caulinares entre 10,1 e $15 \mathrm{~cm}, 13,4 \%$ (Figura 6b).

\section{DISCUSSÃO}

Em um estudo de comunidades de áreas de caatinga no semiárido baiano, Ramalho (2008) demonstrou densidade absoluta de 46 ind. ha ${ }^{-1}$ e frequência absoluta de $32 \%$ para Cereus jamacaru, 

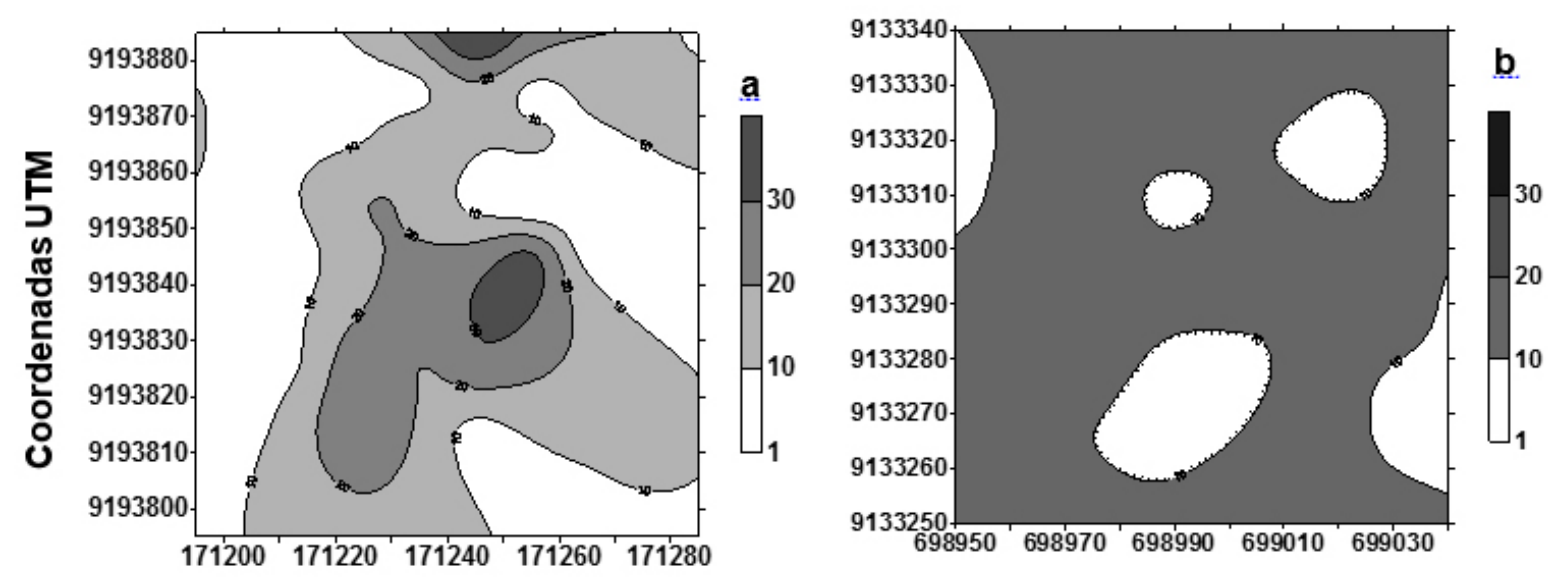

Coordenadas UTM

FIGURA 6. Mapa de isolinhas de distribuição espacial das classes de diâmetro de Cereus jamacaru autor em duas áreas de caatinga em estágio sucessional intermediário. (a) Área de Campina Grande DC, (b) Área de Monteiro - PB.

FIGURE 6. Contoured map of the spatial distribution of diameter classes of Cereus jamacaru DC. in two areas of Caatinga in intermediate successional stage. (a) Area of Campina Grande. (b) Area of Monteiro - PB.

denotando que a espécie não apresenta alta densidade em formações vegetais secundárias.

A estrutura hipsométrica da espécie demonstrou fase de estabelecimento na área de Campina Grande. No entanto, em Monteiro, os espécimes apresentaram estrutura típica de populações estabelecidas com sinais de perturbação no ciclo de vida da espécie, caracterizado pela ausência de indivíduos regenerantes. Isso pode, a médio prazo, significar o comprometimento da sobrevivência da espécie na região. Segundo Howe e Westley (1997), para a reprodução implicar na formação de indivíduos regenerantes, as sementes necessitam ser dispersas para locais favoráveis ao seu crescimento. De modo análogo, o sucesso do recrutamento requer que as sementes sejam dispersas para locais distantes da geração parental, pois nas adjacências desta, a competição é intensificada e os riscos de morte por patógenos ou herbívoros é alta.

O estudo da distribuição dos indivíduos de Cereus jamacaru por meio de histogramas de classes de diâmetro permitiu inferir sobre indicadores temporais das populações (SCOLFORO et al., 1998; PULZ et al., 1999), bem como indicou perturbação para a espécie na área de Campina Grande. Foi possível detectar a existência de perturbações sofridas pela espécie em algum ponto do ciclo de sua vida, e, por conseguinte, fazem-se necessárias ações estratégicas para a conservação da espécie na região a médio prazo. Esse padrão estrutural é preocupante, uma vez que uma das características das cactáceas é a participação obrigatória de dispersores e polinizadores de seus propágulos que as tornam vulneráveis a perturbações ambientais, assim como as distribuições geográficas restritas, ciclo de vida longo e baixa taxas de crescimento o individual (GODÍNEZ-ÁLVAREZ et al., 2003)

Nas duas áreas estudadas, os espécimes apresentaram tendência ao agrupamento e à agregação e, segundo Janzen (1976), este fato é comum para espécies que possuem síndromes de dispersão zoocórica e autocórica. Neste sentido, Quirino (2006) comentou que a dispersão de Cereus jamacaru é ornitocórica. Ao que parece, a fauna ornitófila, ao se alimentar das sementes, excretam nas imediações das plantas matrizes, levando desse modo a uma configuração espacial que se distancia da uniformidade e aleatoriedade (THOMAS; KUNIN, 1999). A espécie apresentou padrão de distribuição similar ao de outros representantes da família Cactacteae (GOMES et al., 2014), aparentando ser um típico padrão de distribuição para a família.

Segundo Legendre e Fortin (1989), os seres vivos em geral tendem a se distribuir de forma agrupada, devido ao ambiente ser estruturado espacialmente por várias formas de produção de energia, gerando, desta forma, processos irregulares de disponibilização de recursos. 
Santos (2009) aponta para a necessidade de ações de conservação no Brasil, uma vez que a região leste do país está entre os três maiores centros de diversidade de cactáceas do mundo. As medidas que podem ser adotadas no âmbito da preservação da espécie na região vão desde a manutenção dos dispersores naturais até a coleta e beneficiamento de material propagativo da espécie, para servir de subsídio para o recrutamento de novos indivíduos. Tais medidas mobilizariam todos os atores envolvidos, incluindo também organizações governamentais e não governamentais que passariam inicialmente por processos de sensibilização sobre a importância dessas ações.

\section{CONCLUSÃO}

Os indivíduos de Cereus jamacaru demonstram características estruturais similares para densidade absoluta e padrão de agregação e diferentes padrões de crescimento. Em ambas as áreas de caatinga, os indivíduos apresentam tendência ao agrupamento, todavia, a população de Monteiro - PB apresenta risco de não se perpetuar no ambiente ao longo dos anos.

Fatores como as condições ambientais, disponibilidade de recursos, distúrbios naturais ou antrópicos, influenciam a estrutura e a dinâmica vegetal e, por conseguinte, as populações de C. jamacaru.

\section{REFERÊNCIAS BIBLIOGRÁFICAS}

ANDERSON, E. F. The cactus famlily. Portland: Timbre Press, 2001. 777 p.

BARBOSA, A. S. Estrutura da vegetação e distribuição espacial de Cactaceae em áreas de caatinga do semiárido paraibano. 2011. 166 f. (Dissertação de Mestrado em Agronomia) - Universidade Federal da Paraíba, Areia, 2011.

BOYLE, T. H.; ANDERSON, E. Biodiversity and conservation. In: NOBER, P. S. (ed.). Cacti: biology and uses. Los Angeles: University of California, 2002. p. 125-141.

CAVALCANTI, N. B.; RESENDE, G. M. Utilização do Xiquexique (Pilosocereus gounellei (A. Weber ex K. Schum.) Bly. ex Rowl) na alimentação dos animais. [s. d.]. Disponível em: $<$ www.infoteca.cnptia.embrapa.br/bitstream/CPATSA/35184/.../OPB1201.pdf> Acesso em: 18 mar. 2010. CARLSON, R. E.; FOLEY, T. A. The parameter $\mathrm{R}^{2}$ in multiquadric interpolation. Computers \& Mathematics with Applications, New York, v. 21, n. 9, p. 29-42, 1991.

COREL DRAW $^{\circledR}$ v. X4. Otawa: Corel Corporation, 2012.

COMPANHIA DE PESQUISA DE RECURSOS MINERAIS. Diagnóstico do município de Campina Grande, estado da Paraíba. Recife: CPRM/PRODEEM, 2005a. 20 p.

CARLSON, R. E.; Diagnóstico do município de Monteiro, estado da Paraíba. Recife: CPRM/ PRODEEM, 2005b, $11 \mathrm{p}$.

EMBRAPA. Centro Nacional de Pesquisa de Solos. Sistema brasileiro de classificação de solos. Brasília: Embrapa Produção de Informação; Rio de Janeiro: Embrapa Solos, 2006. 306 p.

GOMES, V. G. N.; QUIRINO, Z. G. M.; ARAUJO, H. F. P. Frugivory and seed dispersal by birds in Cereus jamacaru DC. ssp. jamacaru (Cactaceae) in the Caatinga of Northeastern Brazil. Brazilian Journal of Biology, São Carlos, v. 74, n. 1, p. 32-40, 2014.

GUERRERO, P. C.; DURÁN, P.; WALTER, H. Patrones biogeográficos de las cactáceas endémicas de Chile. In: CONGRESSO NACIONAL DE BOTÂNICA, 59., 2008, Natal, RN. Natal: Imagem Gráfica, 2008. p. 492-494.

HERNÁNDEZ, H. M.; BÁRCENAS, R. T. Endangered cacti in the Chiuahuan Desert: I. distribution patterns. Conservation Biology, Washington, v. 9, n. 5, p. 1176-1188, 1995.

HOWE, H. F.; WESTLEY, L. C. Ecology of pollination and seed dispersal. In: CRAWLEY, M. J. (Ed.). Plant Ecology. Oxford: Blackwell Science, 1997. p. 262-282.

HUNT, D.; TAYLOR, N. P.; CHARLES, G. The new cactus lexicon. Milborne: DH Books, 2006. v. 2. IBGE. Manual técnico da vegetação brasileira. Rio de Janeiro: IBGE, 2012.

JANZEN, D. H. Why bamboos take so long to flower. Annual Review of Ecology and Systematics, Palo Alto, v. 7, p. 347-391, 1976.

LEGENDRE, P.; FORTIN, M. J. Spatial pattern and ecological analysis. Vegetation, Knivsta, v. 80, 
P. 107-138, 1989.

MISSOURI BOTANICAL GARDEN. Base de dados Tropicos. Disponível em: $<$ http://www.tropicos.org/> Acesso em: 21 jun. 2011.

MOURELLE, C.; EZCURRA, E. Differentiation diversity of Argentine cacti and its relationship to environmental factors. Journal of Vegetation Science, Knivsta, v. 8, p. 547-558, 1997.

MULlER-DOMBOIS, D.; ELLENBERG, H. Aims and methods of vegetation ecology. New York: Wiley, 1974. 574 p.

NYFFELER, R. Phylogenetic relationships in the cactus family (Cactaceae) based on evidence form trnK/ $m a t \mathrm{~K}$ and $t r n \mathrm{~F}$ sequences. American Journal of Botany, Lancaster, n. 89, p. 312-326, 2002.

PULZ, F. A. et al. Acuracidade da predição da distribuição diamétrica de uma floresta inequiânea com a matriz de transição. Cerne, Lavras, v. 5, p. 1-14, 1999.

QUIRINO, Z. G. M. Fenologia, síndromes de polinização e dispersão e recursos florais de uma comunidade de caatinga no cariri paraibano. 2006. $128 \mathrm{f}$. Tese (Doutorado em Biologia Vegetal) - Universidade Federal de Pernambuco, Recife, 2006.

RAMALHO, C. I. Estrutura da vegetação e distribuição espacial do licuri (Syagrus coronata (Mart) Becc.) em dois municípios do centro norte da Bahia, Brasil. 2008. 168 f. Tese (Doutorado em Agronomia) - Centro de Ciências Agrárias, Universidade Federal da Paraíba, Areia, 2008.

REINALDO, L. R. L. R.; SILVA, E. A. Caracterização e classificação de solos da mesorregião agreste paraibana, microrregião de Campina Grande. In: REUNIÃO ANUAL DA SBPC, 58., 2006, Florianópolis, SC. Anais... Florianópolis, 2006.

RIBEIRO, E. M. S. Influência de perturbações antrópicas sobre populações de cactáceas em áreas de caatinga. 2011. 55f. Dissertação de Mestrado (Biologia Vegetal) - Universidade Federal de Pernambuco, Recife, 2011.

RODAL, M. J. N.; SAMPAIO, E. V. S.; FIGUEIREDO, M. A. Manual sobre métodos de estudo florístico e fitossociológico: ecossistema caatinga. Brasília: Sociedade Botânica do Brasil, 1992. 24 p.

SANTOS, M. R. Influência de variáveis ambientais e espaciais na distribuição geográfica da família cactaceae no leste do Brasil. 2009. 76 f. Dissertação (Mestrado em Ecologia Aplicada) Universidade Federal de Lavras, Lavras, 2009.

SCOLFORO, J. R. S.; PULZ, F. A.; MELLO, J. M. Modelagem da produção, idade das florestas nativas, distribuição espacial das espécies e a análise estrutural. In: (Org.). Manejo Florestal. Lavras: UFLA/ FAEPE, 1998. p. 189-245.

SOUZA, V. C.; LORENZI, H. Botânica sistemática: guia ilustrado para identificação das famílias de Angiospermas da flora brasileira. Nova Odessa: Instituto Plantarum, 2005. 639 p.

SURFER ${ }^{\circledR}$. Colorado: Golden software, 2008.

TAYLOR, N. P.; ZAPPI, D. C. Cacti of eastern Brazil. Richmond: The Royal Botanic Gardens, 2004. 499 p.

THOMAS, C. D. E.; KUNIN, W. E. The spatial structure of populations. Journal of Animal Ecology, Oxford, v. 68, p. 647-657, 1999.

GODÍNEZ-ÁlVAREZ, H. V. T.; VALVERDE, T.; ORTEGA-BAES, P. Demographic trends in the Cactaceae. Botanical Review, New York, v. 69, p. 173-203. 2006. 